\title{
Giant thoracic ganglioneuroma with lymph node infiltration of a child: a case report
}

\author{
Mathula Hettiarachchi ${ }^{1,2^{*}}$ D, Chanuka Dharmadasa ${ }^{3}$, Ashoka Wijeyananda ${ }^{4}$, Kapila Hettiarachchi ${ }^{4}$ and \\ Bandula Samarasinghe ${ }^{5}$
}

\begin{abstract}
Background: Ganglioneuroma is a rare neurogenic tumor which could grow into massive size in the thorax with minimal or no symptoms. Resection of these tumors will be challenging in view of large size and vascular encasement. Here, we report resection of a thoracic ganglioneuroma in a child with infiltration of spinal canal and lymph node metastasis, which is a very rare occurrence.

Case presentation: A 3-year and 10-month-old girl presented with a left side thoracic mass with minimal symptoms and signs. After the initial imaging with ultrasound scan and cross-sectional imaging, she had a true cut biopsy of the lesion confirming the diagnosis of ganglioneuroma. On exploration, she had a paraspinal thoracic mass extending from level of arch of aorta down to diaphragm with evidence of tumor infiltration of the intercostal spaces and spinal canal in multiple vertebral levels. Pathologically, she had infiltrated lymph nodes which were encircling the lower thoracic aorta. The tumor was resected successfully, and she recovered with a good functional status.

Conclusion: Thoracic ganglioneuroma has a good prognosis after complete resection even with spinal canal and lymph node infiltration. The resection could be arduous in case of massive size and vascular encasement; hence, benefit versus risk of complete resection should be carefully weighed.
\end{abstract}

Keywords: Thoracic ganglioneuroma, Lymph node, Child, Thoracic mass, Neurogenic tumors

\section{Background}

Ganglioneuroma (GN) is a rare tumor arising from neural crest cells of sympathetic ganglia or adrenal medulla. Because of the slow growth and benign nature of these tumors, they could develop into very large tumors especially in the thorax without being clinically evident [1]. Lymph node metastasis in GN is a very rare occurrence which, according to current evidence, has no effect on long-term prognosis.

We present a case report of a 3-year and 10-monthold girl who had a thoracic GN with lymph node

\footnotetext{
* Correspondence: drmathula@yahoo.co.uk

${ }^{1}$ Faculty of Medicine, University of Peradeniya, Peradeniya, Sri Lanka 2Department of Paediatric Surgery, Sirimavo Bandaranaike Specialised Children's Hospital, Peradeniya, Sri Lanka

Full list of author information is available at the end of the article
}

infiltration and spinal canal infiltration treated successfully with near complete resection.

\section{Case presentation}

A 3-year and10-month-old girl was found to have a large left thoracic mass while being investigated for tachypnoea and prominent left hemithorax as noticed by her mother. She was assessed with a chest X-ray, contrastenhanced computerized tomography (CT) (Fig. 1) and magnetic resonance imaging (MRI) (Fig. 2) which revealed a large left thoracic tumor with extension into spinal canal. Spinal canal involvement was less than onethird of the diameter without evidence of neurological sequelae.

The tumor was shown to be extending from the level of T3 to T11 in the thorax, with external compression of the left main stem bronchus though there was no 


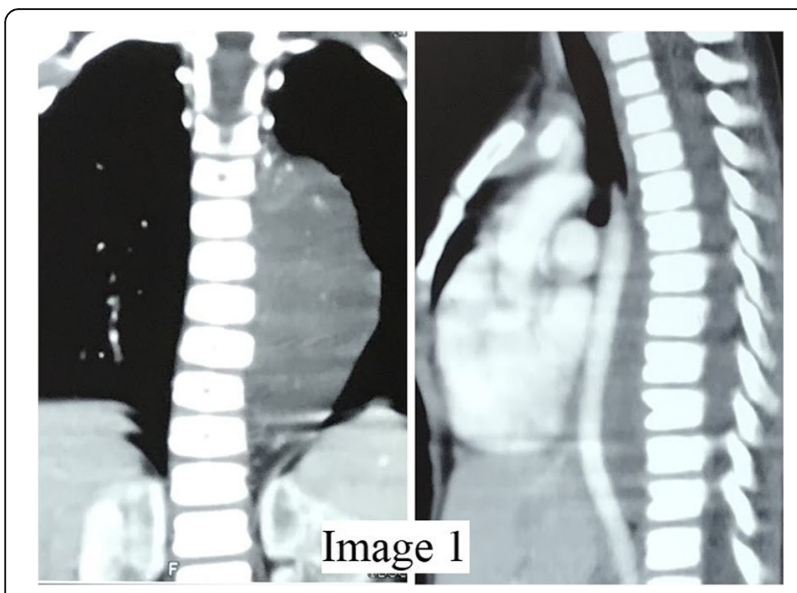

Fig. 1 Contrast-enhanced CT of the child showing the extent of left thoracic mass with specks of calcifications. In the lateral view, the aorta is displaced anteriorly by the encircling mass

evidence of infiltration. Even though image defined risk factors were evident with the presence of partial encasement of the aorta and spinal canal involvement, surgical resection was contemplated due to benign nature of the disease and compression of the mediastinal structures by the mass.

She underwent a trucut biopsy of the lesion which showed features of a GN; hence, primary resection of the tumor was planned.

During the surgery, tumor was found to extend from the level of arch of the aorta down to the diaphragm. Laterally, it was seen extending through 5 th to 9th intercostal spaces. Lower part of the tumor was encircling nearly $60 \%$ of the thoracic aorta, with a separate lesion over the right thorax which was in continuity with the main tumor. This separate lesion on the right thorax

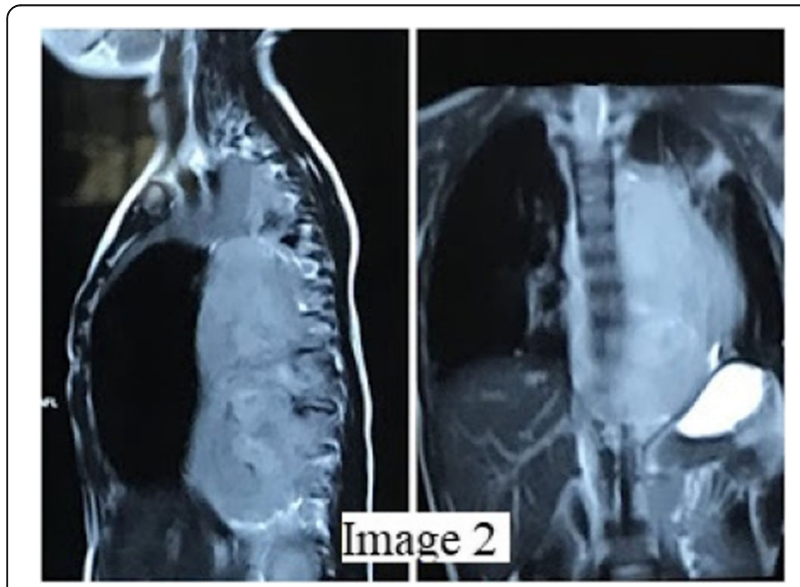

Fig. $2 \mathrm{MRI}$ scan of the thorax and the spine showing the extent of the lesion and spinal canal infiltration was found to be a lymph node mass infiltrated with the tumor.

The tumor was initially accessed through 5 th intercostal space. Due to the large size of the tumor, lower part was accessed through 8th intercostal space, which was opened through the same skin incision. Posterolateral plane of the tumor was initially opened up by sharp and blunt dissection. The tumor was shaved off the intervertebral and intercostal spaces which were infiltrated by the mass. After adequate mobilization, the tumor was transected in the middle for easy handling of the lower part. Once the tumor was free from the bony structures, traction on the tumor facilitated dissection around the aorta. Closely related lymph node mass was separately excised after the main mass was excised. Remnants of the tumor in the intercostal spaces were excised piecemeal after the main tumor was resected (Fig. 3). Anterior medullary artery arising from lower thoracic aorta was not encountered with active surveillance during resection.

Post-operatively, she developed collapse of the left main bronchus for which she had to be on ventilator for 2 weeks.

Histology of the resected tumor confirmed the diagnosis of a ganglioneuroma with mediastinal lymph node infiltration which were encircling the lower thoracic aorta (Figs. 4 and 5).

She did not need neurosurgical intervention based on the interval post-operative MRI scan which showed shrunken intraspinal lesion without significant spinal cord or nerve compression. She was followed up with a chest X-ray which did not show evidence of gross macroscopic recurrence up to the last review at 6 months of resection. She needs to be followed up further with cross-sectional imaging preferably a MRI scan to

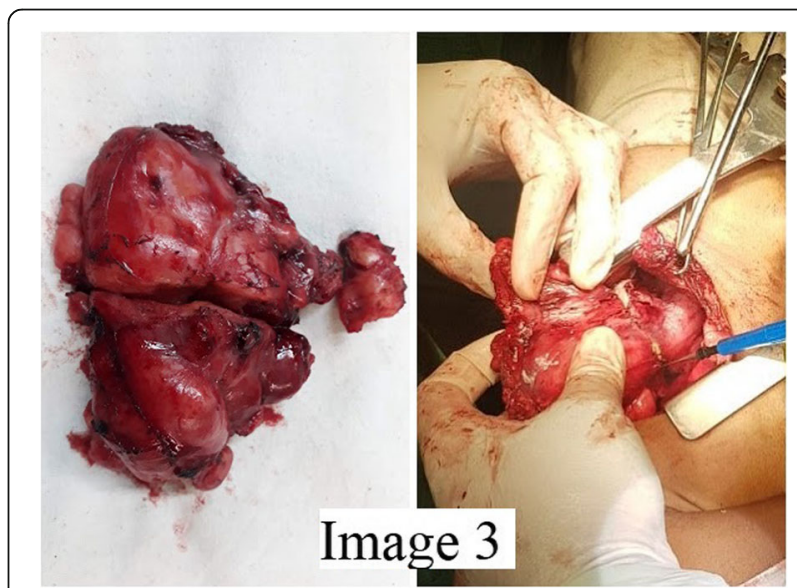

Fig. 3 Intraoperative view of the tumor being transected in the middle and the resected mass showing the attached infiltrated lymph node 


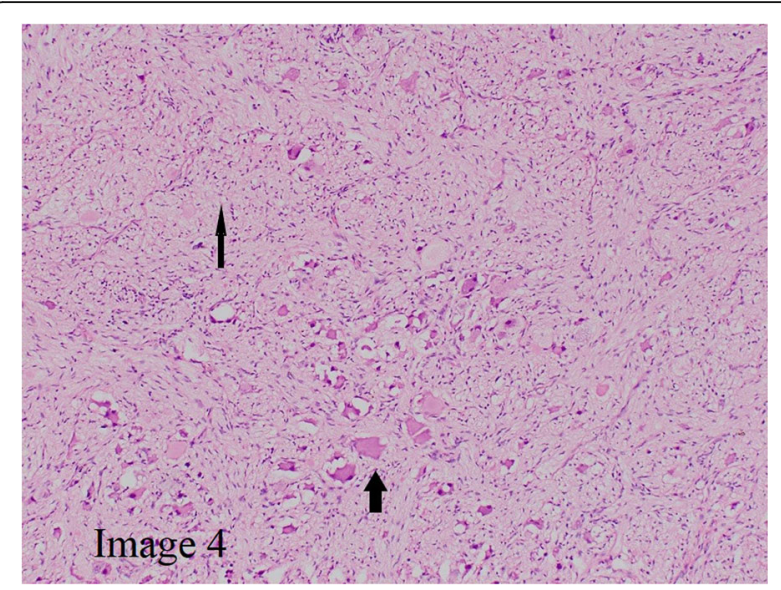

Fig. 4 Hematoxylin and eosin stain showing ganglioneuroma with mature ganglion cells and Schwannian rich stroma (thick arrow indicates a mature ganglion cell, and thin arrow indicates the schwannian rich stroma)

assess the mediastinal nodal status and intraspinal lesion.

\section{Discussion}

GN is a benign tumor of neural crest cell origin which is mostly encountered in the mediastinum or retroperitoneum. It belongs to a group of neurogenic tumors which accounts for $85-90 \%$ of pediatric posterior mediastinal masses [2].

Symptoms could arise from metabolic activity of GN or due to local pressure effects of expanding tumor but neither was there in our patient. Even though there was spinal canal infiltration, neurological sequelae were not evident.

In the $\mathrm{CT}, \mathrm{GN}$ appears as a well-defined mass with specks of calcification in $20-42 \%$ of cases [3]. MRI will

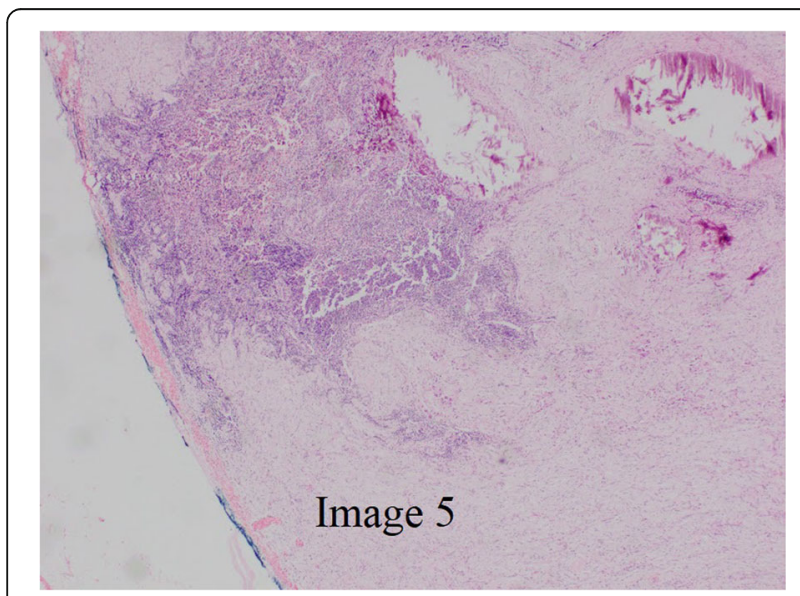

Fig. 5 Hematoxylin and eosin stain of the lymph node showing lymphoid tissue, calcified foci, and ganglioneuromatous tissue be useful to define intraspinal extension in $\mathrm{GN}$ which occurs due to its paraspinal location.

According to the International Neuroblastic Classification system, GN is classified as Schwannian stroma rich tumor having a favorable prognosis [4]. Histologically, GN are mainly composed of mature ganglion cells, Schwannian stroma, and nerve fibers (Fig. 4), but diagnosis should not be made when neuroblastic elements or mitotic figures are present in the histology [3]. In our patient, there were no neuroblastic components or mitotic figures in the histology, but there was evidence of lymph node metastases (Fig. 5).

Even though GN is typically a benign tumor, lymph node metastases could occur. In one study, 2 out of 49 patients with GN demonstrated to have lymph node metastases, but both were abdominal in origin [5]. In another study, 2 out of 11 patients with GN demonstrated to have lymph node metastases, but only 1 of them had thoracic GN [6]. Lymph node metastasis indicates that the tumor may have arose as a neuroblastoma (NBL) or ganglioneuroblastoma (GNB) which has metastasized and subsequently differentiated into mature GN [5]. However, the prognosis of GN is excellent even with lymph nodes or soft tissue metastases [5].

The definitive treatment of ganglioneuroma is surgical resection either by open surgery or by minimal access surgery when feasible [4]. Prognosis is highly favorable in GN compared to NBL or GNB. Recurrence risk of GN after resection is minimal compared to other neurogenic tumors, even if complete resection was not undertaken [5].

At 6 months review, there was no gross evidence of recurrence in our patient and she is having normal functional capacity and exercise tolerance. However, she was showing musculoskeletal disability on the left side due to morbidity of a rib spreading thoracotomy compared to right side.

\section{Conclusions}

Prognosis of thoracic ganglioneuroma is excellent after complete resection despite spinal canal infiltration and lymph node metastasis.

Resection of GN could be arduous due to its massive size and vascular encasement; hence, risk of resection should be carefully weighed against the benefit of complete resection.

\section{Abbreviations \\ GN: Ganglioneuroma; CT: Computerized tomography; MRI: Magnetic resonance imaging; NBL: Neuroblastoma; GNB: Ganglioneuroblastoma}

\section{Acknowledgements}

We would like to express our sincere gratitude to the patient for giving the consent to present this case in this paper. We would like to appreciate Dr. Sulochana Wijethunga, Consultant Pathologist, Teaching Hospital Peradeniya, 
Sri Lanka, for supplying us with the digital images of the histopathology slides.

\section{Authors' contributions}

$\mathrm{MH}, \mathrm{CD}, \mathrm{AW}, \mathrm{KH}$, and $\mathrm{BS}$ were responsible for delivering the patient care. $\mathrm{MH}$ wrote the case and the manuscript. Editing and supervision were done by $\mathrm{MH}, \mathrm{CD}$, and BS. All authors revised, read, and approved the final manuscript.

\section{Funding}

No funding or grant support was available for this case report.

\section{Availability of data and materials}

Relevant data and materials are available for review if needed.

\section{Ethics approval and consent to participate}

Not applicable.

\section{Consent for publication}

Written informed consent was obtained from a parent of the patient for publication of this case report and accompanying images. A copy of the consent form is available for review.

\section{Competing interests}

The authors declare that they have no competing interests.

\section{Author details}

${ }^{1}$ Faculty of Medicine, University of Peradeniya, Peradeniya, Sri Lanka. ${ }^{2}$ Department of Paediatric Surgery, Sirimavo Bandaranaike Specialised Children's Hospital, Peradeniya, Sri Lanka. ${ }^{3}$ Department of Pathology, Sirimavo Bandaranaike Specialised Children's Hospital, Peradeniya, Sri Lanka. ${ }^{4}$ Department of Anaesthesia, Sirimavo Bandaranaike Specialised Children's Hospital, Peradeniya, Sri Lanka. ${ }^{5}$ Department of Surgery, University Surgical Unit, Teaching Hospital, Peradeniya, Sri Lanka.

Received: 16 August 2020 Accepted: 29 November 2020

Published online: 22 December 2020

\section{References}

1. Schulman H, Laufer L, Barki Y, Philip M, Mares AJ, Maor E, Hertzanu Y. Ganglioneuroma: an 'incidentaloma' of childhood. Eur Radiol. 1998;8(4):5824.

2. Franco A, Mody NS, Meza MP. Imaging evaluation of pediatric mediastinal masses. Radiol Clin. 2005;43(2):325-53.

3. Lonergan GJ, Schwab CM, Suarez ES, Carlson CL. From the archives of the AFIP: neuroblastoma, ganglioneuroblastoma, and ganglioneuroma: radiologic-pathologic correlation. Radiographics. 2002;22(4):911-34.

4. Shimada H, Umehara S, Monobe Y, Hachitanda Y, Nakagawa A, Goto S, Gerbing RB, Stram DO, Lukens JN, Matthay KK. International neuroblastoma pathology classification for prognostic evaluation of patients with peripheral neuroblastic tumors: a report from the Children's Cancer Group. Cancer. 2001;92(9):2451-61.

5. Geoerger B, Hero B, Harms D, Grebe J, Scheidhauer K, Berthold F. Metabolic activity and clinical features of primary ganglioneuromas. Cancer. 2001; 91(10):1905-13.

6. Ann Hayes F, Green AA, Rao BN. Clinical manifestations of ganglioneuroma. Cancer. 1989;63(6):1211-4.

\section{Publisher's Note}

Springer Nature remains neutral with regard to jurisdictional claims in published maps and institutional affiliations.

\section{Submit your manuscript to a SpringerOpen ${ }^{\circ}$ journal and benefit from:}

- Convenient online submission

- Rigorous peer review

- Open access: articles freely available online

- High visibility within the field

- Retaining the copyright to your article 